\title{
Nanotheranostics
}

2017; 1(2): 217-231. doi: 10.7150/ntno.19158

Research Pape

\section{Improving Brain Delivery of Biomolecules via BBB Modulation in Mouse and Rat: Detection using MRI, NIRF, and Mass Spectrometry}

Kavisha R. Ulapane ${ }^{1,2 *}$, Ngoc On ${ }^{3 *}$, Paul Kiptoo ${ }^{2}$, Todd D. Williams ${ }^{4}$, Donald W. Miller ${ }^{3}$, and Teruna Siahaan $1,2 \bowtie$

1. Department of Chemistry, The University of Kansas, Lawrence, KS 66047, USA;

2. Department of Pharmaceutical Chemistry, The University of Kansas, Lawrence, KS 66047, USA;

3. Department of Pharmacology and Therapeutics, University of Manitoba, Winnipeg, Manitoba, Canada;

4. Mass Spectrometry Laboratory, The University of Kansas, Lawrence, KS 66047, USA.

* Both KRU and NO contributed equally to this work.

$\triangle$ Corresponding author: Dr. Teruna J. Siahaan, Department of Pharmaceutical Chemistry, The University of Kansas, Lawrence, KS 66047, USA Phone: 785-864-7327 Fax: 785-864-5736 E-mail: siahaan@ku.edu

(C) Ivyspring International Publisher. This is an open access article distributed under the terms of the Creative Commons Attribution (CC BY-NC) license (https://creativecommons.org/licenses/by-nc/4.0/). See http://ivyspring.com/terms for full terms and conditions

Received: 2017.01.13; Accepted: 2017.05.02; Published: 2017.06.08

\begin{abstract}
There is an urgent need to develop new and alternative methods to deliver functional biomolecules to the brain for diagnosis and treatment of brain diseases. The goal of this study was to evaluate the activity of blood-brain barrier (BBB) modulators (i.e., HAV and ADT peptides) to deliver functional biomolecules (i.e., galbumin, IRdye $800 \mathrm{cw}-\mathrm{cLABL}$, and cIBR7) to the brains of mice and rats. HAV6, cHAVc3, and ADTC5 peptides but not HAV4 peptide significantly enhanced the brain delivery of $65 \mathrm{kDa}$ galbumin compared to control in Balb/c mice as quantified by magnetic resonance imaging (MRI). Ten-minute pretreatment with ADTC5 peptide still significantly increased brain delivery of galbumin; however, no enhancement was observed after 10-min pretreatment with HAV6. There was no enhancement of galbumin deposition following 40-min pretreatment with ADTC5 or HAV6, suggesting a short duration of the BBB opening for large molecules. ADTC5 peptide also improved the brain delivery of IRdye $800 \mathrm{cw}$-cLABL peptide about 3.5-fold compared to control in Balb/c mice as detected by near infrared fluorescence (NIRF). The BBB modulator activity of ADTC5 to deliver cIBR7 peptide was also evaluated in vivo using Sprague-Dawley rats. The amount of clBR7 in the brain was detected by LC-MS/MS. ADTC5 peptide enhanced the delivery of clBR7 peptide into rat brain about 4-fold compared to control and the intact clBR7 can be efficiently extracted and detected in rat brain. In conclusion, HAV and ADT peptides enhance the brain delivery of functional peptides (e.g., cLABL and cIBR7) and protein (e.g., $65 \mathrm{kDa}$ galbumin) in two animal models, and the duration of the BBB opening for a large molecule (e.g., galbumin) was short.
\end{abstract}

Key words: blood-brain barrier, BBB, brain delivery, cadherin peptides, HAV peptide, ADT peptide, paracellular diffusion, tight junction, adherens junction, BBB modulation.

\section{Introduction}

There is an urgent need to develop new and alternative methods to deliver molecules to the brain for diagnosis and treatment of brain diseases (i.e., Parkinson's, Alzheimer's, multiple sclerosis, brain tumors) [1, 2]. Furthermore, approaches that can safely deliver molecules to the brain can also provide opportunities to probe brain functions at the cellular and molecular levels using selected imaging and cellular signaling molecules. The protective function of the BBB selectively restricts molecules from 
entering the brain; however, under pathological conditions, the BBB can also restrict the accumulation of drugs to the brain. Numerous invasive methods have been developed to directly deliver or sample molecules to or from the brain, respectively; these methods include (a) brain microdialysis [3], (b) intracerebral implantation [4], and (c) intraventricular delivery [5]. However, these methods can be problematic for patients, and these invasive approaches can cause damage in the surrounding brain tissues. Alternatively, various non-invasive approaches have been developed to improve brain delivery of molecules, including the use of (a) prodrug technology, (b) efflux pump inhibitors, (c) receptor-mediated transport, (d) osmotic agents, and (e) BBB modulators; however, in general, the success of these methods has been limited [1, 2, 6, 7].

One way to deliver drug and diagnostic molecules to the brain is via the transcellular pathway, allowing the molecule to diffuse through the membranes of the BBB endothelial cells. Traditionally, changing the physicochemical properties of the drug was done to improve its brain delivery via the transcellular pathway. However, changing the drug structure may alter its biological activity. As an alternative, several prodrug methods have been developed to transiently change the physicochemical properties of the drug, permitting greater transcellular BBB penetration [1]. Although the physicochemical properties of the drug can be changed to improve its membrane partition for transcellular passive diffusion, the presence of efflux pumps on the BBB can prevent the drug molecule from crossing the BBB cell membranes. Inhibitors have been designed to block the activity of efflux pumps in allowing the drug to cross the BBB [1]. Receptor-mediated transcytosis has also been explored for improving brain delivery of molecules. For example, the high expression of transferrin receptors on the luminal side of brain capillary endothelial cells has been exploited as a carrier of drugs/macromolecules across the BBB, and has shown promise in pre-clinical animal models $[2,8]$.

An alternative approach to deliver molecules across the BBB is via the paracellular pathway by increasing the porosity of the intercellular junctions. Under normal conditions, the penetration of molecules through this pathway is limited by the presence of the tight intercellular junctions. Therefore, modulation of these junctions is needed to improve paracellular passive diffusion of molecules. One successful method for enhancing paraceullar delivery across the BBB that has been used in the clinic to deliver anticancer drugs to brain tumor patients is hyperosmotic agents such as mannitol. In this case, administration of osmotic agents is used to produce a hypertonic environment within the brain vasculature, causing shrinkage of endothelial cells of the BBB and allowing opening of the tight junctions for molecules to cross via paracellular pathways [9]. The positive results from the osmotic method have stimulated investigation of other methods to transiently modulate the integrity of the BBB to enhance molecule delivery via the paracellular pathway. Several molecules have been developed to modulate the intercellular junctions of the $\mathrm{BBB}$, including bradykinin derivatives and inhibitors of cell-cell adhesion proteins (i.e., occludins, claudins, cadherins) [10-13].

Our group have designed and utilized cadherin peptides called HAV (His-Ala-Val) [14] and ADT (Ala-Asp-Thr) [15] peptides derived from the extracellular domain-1 (EC1) of E-cadherin (Table 1) to modulate the intercellular junctions of the BBB and improve paracellular penetration of functional biomolecules (i.e., peptides and proteins). Previously, HAV and ADT peptides have been shown to enhance the in vivo brain delivery of small-to-large marker molecules (e.g., ${ }^{14} \mathrm{C}$-mannitol, gadopentetic acid, and $25 \mathrm{kDa}$ polyethylene glycols (PEG)) in mice and rats [16-20]. However, the utility of HAV and ADT peptides in enhancing in vivo brain delivery of proteins and peptides has not yet been evaluated. Thus, the aim of this work was to evaluate the ability of cadherin peptides to enhance brain delivery of galbumin, IRdye $800 \mathrm{cw}-\mathrm{cLABL}$ peptide, and unlabeled cIBR7 peptide.

Table 1. Peptide Names and Peptide Sequences

\begin{tabular}{lll}
\hline $\begin{array}{l}\text { Peptide } \\
\text { Name }\end{array}$ & Sequence & Peptide Origin \\
\hline HAV6 & Ac-SHAVSS-NH & \\
HAV4 & Ac-SHAVAS-NH & \\
cHAVc3 & Cyclo(1,6)Ac-CSHAVC-NH & EC1 of E-cadherin \\
ADTC5 & Cyclo(1,7)Ac-CDTPPVC-NH & EC1 of E-cadherin \\
cLABL & Eyclo(1,12)PenITDGEATDSGC & I-domain of LFA-1 \\
cIBR7 & Cyclo(1,8)CPRGGSVC & D1 of ICAM-1 \\
IS & Cyclo(1,8)CPRGGSIC & Ile7 mutant of cIBR7 \\
\hline
\end{tabular}

In this study, four different cadherin peptides (HAV4, HAV6, cHAVc3, ADTC5) were evaluated in their effectiveness for delivering a large protein, galbumin, a gadolinium-labeled albumin magnetic resonance imaging contrast agent, into the brains of Balb/c mice. Both the extent and duration of BBB modulation by HAV6 or ADTC5 peptides was evaluated. Galbumin is a gadolinium-labeled albumin that can be detected using magnetic resonance imaging (MRI) due to the increase in $\mathrm{T}_{1}$ relaxation rate $\left(1 / T_{1}\right)$ to produce a positive image contrast in the 
brain [21]. Due to its physicochemical properties (i.e., size, hydrogen binding potential, and hydrophilicity), galbumin cannot cross the BBB via the transcellular or paracellular pathways. Therefore, it is a good model molecule to test the activity of cadherin peptides to enhance brain delivery of protein via the paracellular pathway due to the increase in BBB paracellular porosity.

Secondly, the brain delivery of IRdye800cw-cLABL peptide was accomplished using ADTC5 peptide in Balb/c mice. cLABL peptide was derived from the I-domain sequence of lymphocyte function-associated antigen-1 (LFA-1) receptors found on the surface of $\mathrm{T}$ cells. The cLABL peptide binds to and is internalized by intercellular adhesion molecule-1 (ICAM-1) on the surface of vascular endothelial and immune cells [22, 23]. Because ICAM-1 is upregulated during inflammation, the hypothesis is that labeled cLABL peptide can be used to detect changes in ICAM-1 expression in the brain during neuroinflammation. In this study, the brain deposition of cLABL was quantified using near-infrared fluorescence (NIRF) imaging. Although this peptide is smaller than galbumin, it has unfavorable physiochemical properties for crossing the $\mathrm{BBB}$ via the transcellular and paracellular pathways, thus, it is also a good model of peptide delivery to the brain.

Finally, this study was carried out to evaluate the brain delivery of unlabeled cIBR7 peptide using ADTC5 peptide in Sprague-Dawley rats. The aim is to develop a method to extract and detect the unlabeled cIBR7 peptide in the brain to confirm our findings using labeled molecules. Sprague-Dawley rats were used to evaluate the applicability of ADTC5 peptide to enhance delivery of molecules into rat brains in vivo because ADTC5 has not been used in rat model previously. cIBR7 peptide was derived from ICAM-1 sequence and it binds to the I-domain of LFA-1 receptor on immune cells (i.e., T cells); therefore, brain delivery of cIBR7 peptide can detect the presence of immune cells in the brain during the neuroinflammation process [24-26]. Most of our studies to deliver molecules to the brain have been carried out using labeled molecules, and their brain deposition was detected using radioactivity, NIRF imaging, and magnetic resonance imaging (MRI). One possible caveat of using labeled molecules is that the labeled molecule may degrade during delivery. Thus, the detected quantity of the molecule may be due to a combination of the intact molecule and its degraded fragments containing the label. Therefore, unlabeled cIBR7 peptide was delivered using ADTC5 peptide, and brain depositions of cIBR7 and ADTC5 peptides were quantified using tandem mass spectrometry
(LC-MS/MS).

\section{Materials and Methods}

\section{Chemicals, Reagents, and Animals}

Ketamine hydrochloride and xylazine were purchased from MWI Veterinary Supply Co. (Boise, ID) and Lloyd Inc. (Shenandoah, IA), respectively. IRdye800cw-NHS ester was purchased from LiCOR Inc. (Lincoln, NE). Amino acids for peptide synthesis were purchased from Protein Technologies Inc. (Tucson, AZ). Galbumin was purchased from BioPal Inc. (Worcester, MA). All other reagents and chemicals were purchased from Sigma Aldrich Chemical Company (St. Louis, MO). All protocols involving the use of animals were approved by the Institutional Animal Care and Use Committee (IACUC) at The University of Kansas or the University of Manitoba. The animals were maintained in the Animal Care Unit with free access to food and water.

\section{Peptide Synthesis and Purification}

The synthesis of peptides (Table 1) was accomplished using a solid-phase peptide synthesizer. After peptide cleavage from the resin using TFA, the crude peptides were precipitated in cold diethyl ether. In most cases, the crude precipitate showed high concentration of the desired peptide. After purification with semi-preparative HPLC, the isolated peptides had high purity $(>96 \%)$ as determined by analytical HPLC with a C18 column. The formation of a disulfide bond in the cyclic peptide (i.e., cIBR7, ADTC5, cHAVc3, IS) was accomplished by stirring the precursor linear peptide in bicarbonate buffer solution under air oxidation at $\mathrm{pH} 9.0$ in high dilution. The cyclization reaction produced primarily the desired monomer with minor oligomer side-products; the monomer was isolated from the mixture using semi-preparative HPLC. Because cIBR7, IS, and ADTC5 peptides were quantified in the brain by mass spectrometry, their exact mass data are presented in Table 2.

Table 2. Mass Spectrometry Identification of ADTC5, clBR7 and IS.

\begin{tabular}{llll}
\hline Peptide & Exact Mass $(\mathrm{g} / \mathrm{mol})$ & $\begin{array}{l}\text { Accurate Mass } \\
(\mathrm{g} / \mathrm{mol})\end{array}$ & $\begin{array}{l}\text { Mass Error } \\
(\mathrm{ppm})\end{array}$ \\
\hline ADTC5 & 772.293 & 772.298 & 6.5 \\
cIBR7 & 775.315 & 775.316 & 1.3 \\
IS & 789.331 & 789.336 & 6.3 \\
\hline
\end{tabular}

To synthesize IRdye800cw-cLABL, cLABL peptide $(0.86 \mathrm{mg}, 0.71 \mu \mathrm{mol}, \mathrm{MW}=1197.2 \mathrm{~g} / \mathrm{mol})$ and IRdye800cw-NHS ester $(5.0 \mathrm{mg}, 4.2 \mu \mathrm{mol}, \mathrm{MW}=$ 
$1166.2 \mathrm{~g} / \mathrm{mol}$ with $3 \mathrm{Na}^{+}$) were mixed in $1.0 \mathrm{~mL}$ of 0.5 $\mathrm{M}$ potassium phosphate buffer at $\mathrm{pH} 8.5$ followed by stirring overnight in the dark. The reaction progress was monitored by analytical HPLC with a C18 column. After the reaction was completed, IRdye800cw-cLABL (MW $=2248.4$ with $3 \mathrm{Na}^{+}$and 2182.4 without $\mathrm{Na}^{+}$ions) was purified using HPLC with a C18 semi-preparative column. The identity of the pure product was determined using mass spectrometry to give 2182.69 amu for IRdye800cw-cLABL without $\mathrm{Na}^{+}$ions.

\section{In Vivo Delivery of Galbumin in Balb/c Mice and Quantification using MRI}

The effects of cadherin peptides (i.e., HAV4, HAV6, ADTC5, cHAVc3) to enhance brain delivery of $65 \mathrm{kDa}$ galbumin were determined in Balb/c mice. A Bruker Biospect MR spectrometer equipped with 7 tesla/21 cm magnets and $2.5 \times 2.5 \mathrm{~cm}^{2}$ field of view was used to quantify brain depositions of galbumin. After anesthesia, Balb/c mice were placed in the MRI magnet followed by simultaneous delivery of galbumin $(0.6 \mu \mathrm{mol} / \mathrm{kg})$ with the peptide $(0.010$ $\mathrm{mmol} / \mathrm{kg}$ ). The brain was scanned with a series of T1and T2-weighted images before initial delivery of galbumin to determine the brain background images and structural coordinates [17]. Every 3 minutes, T1-weighted images of the brain were collected for up to $51 \mathrm{~min}$ following the injection of galbumin with peptide. The galbumin brain deposition was determined using the intensity of the contrast agent from manually outlined regions of interest (ROI) within the coronal brain slices using Marevisi 3.5 software (Institute for Biodiagnostics, National Research Council, Otawa, Ontario, Canada). Paravision 3.0 software package was used to quantify the increase in image intensity of galbumin in the brain using the following formula for percent change:

[(Post-Gd-DTPA T1-weighted images - Pre-Gd-DTPA T1-weighted images) $\div$ Pre-Gd-DTPA T1-weighted images] $\times 100$

\section{In Vivo Delivery of IRdyeR800cw-cLABL into the Brains of Balb/c Mice and NIRF Detection}

The effect of ADTC5 in enhancing BBB penetration of IRdyeR800cw-cLABL in Balb/c mice was quantified using NIRF imaging as described previously [17]. Briefly, IRdye800cw-cLABL (10 $\mu \mathrm{g} /$ mouse) along with ADTC5 $(0.01 \mathrm{mmol} / \mathrm{kg})$ was delivered into Balb/c mice via tail vein injection. After $20 \mathrm{~min}$, the mice were sacrificed, followed by cardiac perfusion with $10 \%$ formaldehyde solution [17]. The brains were removed and 1-mm coronal slices prepared for ex vivo quantitative analysis using an Odyssey NIRF imaging system (Licor, Lincoln, NE).
The depositions of IRdyeR800cw-cLABL peptide in the brain were quantified by integrating the fluorescence intensities at the ROI. The integrated fluorescence intensities were normalized to the fluorescence from the blood sample at the same time point. The data were presented as the relative fluorescence units $/ \mathrm{mm}^{3}$ of tissue divided by relative fluorescence units $/ \mu \mathrm{L}$ blood.

\section{In vivo Delivery of cIBR7 into the Rat Brain and LC-MS/MS Detection}

ADTC5 peptide was used to deliver cIBR7 in male Sprague-Dawley rats (300-400 g). Rats were anaesthetized with a combination of ketamine (100 $\mathrm{mg} / \mathrm{kg})$ and xylazine $(5 \mathrm{mg} / \mathrm{kg})$ delivered intraperitoneally. A heat lamp was used to maintain the animal's body temperature during the experiment. The jugular vein of the rat was cannulated with a polyethylene catheter (PE 50) containing heparinized saline $(100 \mathrm{IU} / \mathrm{mL})$ through which cIBR7 $(40 \mathrm{mg} / \mathrm{kg})$ and ADTC5 $(30 \mathrm{mg} / \mathrm{kg})$ were administered over $10 \mathrm{~min}$. Then, the rats were sacrificed and a heart-cut was performed followed by perfusion of the brain with saline to remove leftover peptide in the microvessels of endothelial cells of the BBB. The whole brain tissue was isolated and rinsed with saline followed by storage at $-80{ }^{\circ} \mathrm{C}$. In the meantime, blood was collected in tubes containing anticoagulants and centrifuged at $12000 \mathrm{rpm}$ for 10 min. Plasma was obtained and stored at $-80^{\circ} \mathrm{C}$ until further processing. Control studies were done following the same procedure but with the administration of only cIBR7 (without ADTC5). Samples were extracted and analyzed using LC-MS/MS.

\section{Capillary Depletion Method}

Parallel capillary depletion experiments were performed as described by Triguero et al. [27] to ensure that there is no trapping of the delivered molecules in the microvessel endothelial cells. Brain homogenates from rat brains dosed with cIBR7 and ADTC5 were mixed together and divided into two sets. One set of homogenates $(580 \mu \mathrm{L})$ was extracted using the optimized extraction procedure (see below). The other set of brain homogenates was extracted after capillary depletion was performed to remove cerebral vasculature contaminants. In this method, $580 \mu \mathrm{L}$ of $26 \%$ dextran solution was added to $580 \mu \mathrm{L}$ of brain homogenate with IS. The mixture was centrifuged at 5,400 $\mathrm{g}$ for $15 \mathrm{~min}$, and the supernatant was extracted with $1 \mathrm{~mL}$ acetonitrile using the optimized extraction procedure described below. 


\section{LC-MS/MS Method Development and Peptide Extraction and Detection Procedures}

\section{Instrumentation and Chromatographic Conditions for Quantitative LCMS/MS Analysis}

The LC-MS/MS system consisted of an Acquity UPLC system (Waters Corp., Milford, MA) coupled to an electrospray (ESI) ion source and a Quattro Ultima triple quadrupole mass analyzer (Micromass Ltd., Manchester, UK). Separation was performed at room temperature on a Luna UPLC $C_{18}(2.1 \mathrm{~mm} \times 50 \mathrm{~mm}, 5$ $\mu \mathrm{m}$ particle size, $100 \AA$ A; Phenomenex, Inc., Torrance, CA) using a binary gradient mobile phase consisting of solvents (A) $\mathrm{H}_{2} \mathrm{O}$ :formic acid (99.92:0.08) and (B) acetonitrile:formic acid (99.92:0.08). The gradient was carried out as follows: 1\% B (initial-0.5 min), 1-7\% B (3.5 min), $7-18 \%$ B (1 min), $18-50 \%$ B (1 min), 50-80\% B (1 min), 80\% B (2 min), 80-100\% B (1 min) and 100\% B (1 min). A $20-\mu \mathrm{L}$ sample was injected into the mobile phase flowing at a rate of $0.350 \mathrm{~mL} / \mathrm{min}$. Mass spectrometric analysis was conducted in positive ion mode using multiple reaction monitoring (MRM). Nitrogen was used as the desolvation gas and argon gas was used for collision-induced dissociation (CID). Cone voltages for the analytes and collision energies were set at 35-55 V and 35-120 V, respectively. MRM chromatograms were quantified using MassLynx $\mathrm{v}$ 4.1 software (Micromass) for the integration of relevant peak areas.

\section{Preparation of Stock, Standard, and IS Solutions}

ADTC5, cIBR7, and internal standard (IS) were dissolved in nanopure water to prepare $(1 \mathrm{mg} / \mathrm{mL})$ stock solutions, which were stored at $-20^{\circ} \mathrm{C}$ and later diluted for the required working standard solutions. Calibrations of standard brain homogenate samples were prepared as follows. The working standard solutions $(10 \mu \mathrm{L})$ and IS $(10 \mu \mathrm{L}$, target concentration of $200 \mathrm{ng} / \mathrm{mL}$ ) were spiked into $580 \mu \mathrm{L}$ of blank brain homogenates from untreated rats. These were recovered using the extraction procedure given below to yield a linear range from 50 to $500 \mathrm{ng} / \mathrm{mL}(50,100$, $175,250,350,500 \mathrm{ng} / \mathrm{ml}$ ) for the calibration curves. Quality control (QC) samples were prepared in the same way.

\section{Sample Preparation}

\section{Extraction of peptides from brain tissue}

The whole brain was homogenized with $0.25 \%$ acetic acid using a PowerGen 700 tissue homogenizer. $590 \mu \mathrm{L}$ aliquots of rat brain homogenate were spiked with $10 \mu \mathrm{L}$ of IS (target concentration, $200 \mathrm{ng} / \mathrm{mL}$ ). The homogenate was vortexed for $1 \mathrm{~min}$ followed by addition of $1 \mathrm{~mL}$ acetonitrile; the mixture was then vigorously vortexed again for $1 \mathrm{~min}$ followed by centrifugation at $12000 \mathrm{rpm}$. The supernatant was isolated, transferred to a clean tube, and evaporated to dryness using a Savant SpeedVac concentrator. The dry extract was reconstituted in $100 \mu \mathrm{L}$ of water, vortexed for $30 \mathrm{~s}$, and centrifuged for $10 \mathrm{~min}$ at 12000 $\mathrm{rpm}$ to remove residual precipitated proteins.

\section{Extraction of peptides from plasma}

$100 \mu \mathrm{L}$ of rat plasma was spiked with $10 \mu \mathrm{L}$ of IS (target concentration $200 \mathrm{ng} / \mathrm{mL}$ ). The mixture was vortexed for $1 \mathrm{~min}$ followed by addition of $250 \mu \mathrm{L}$ of acetonitrile. The mixture was vigorously vortexed again for $1 \mathrm{~min}$, followed by centrifugation at 12000 $\mathrm{rpm}$. The supernatant was isolated, transferred to a clean tube, and evaporated to dryness using a Savant SpeedVac concentrator. The dry extract was reconstituted in $100 \mu \mathrm{L}$ of water, vortexed for $30 \mathrm{~s}$, and centrifuged for $10 \mathrm{~min}$ at $12000 \mathrm{rpm}$ to remove residual precipitated proteins.

\section{Extraction Recovery}

Recovery and matrix effects of cIBR7 and ADTC5 were tested in the spiked rat brain homogenates at three concentrations 50, 250 and 500 $\mathrm{ng} / \mathrm{mL}$. Extraction recovery of cIBR7 and ADTC5 from brain tissue homogenate was determined by the ratios of peak areas in the post-extraction spiked samples and in pre-extraction spiked samples.

\section{Accuracy, Precision, and Stability}

The intra-day as well as inter-day accuracy and precision were calculated by analyzing replicates of spiked brain homogenates at three concentrations 50 , 250 and $500 \mathrm{ng} / \mathrm{mL}$. The stability of the cIBR7 and ADTC5 peptides in spiked brain homogenates was evaluated under different temperature and storage conditions. Three sets of samples were subjected to (a) room temperature for $10 \mathrm{~h}$, (b) $-20^{\circ} \mathrm{C}$ for $24 \mathrm{~h}$ followed by unassisted thawing at room temperature, and (c) three freeze-thaw cycles between $-20^{\circ} \mathrm{C}$ and room temperature over a $24-\mathrm{h}$ period. The total chromatogram time for one sample was $11 \mathrm{~min}$, and the total duration for a set of samples for the experiment per day was less than $8 \mathrm{~h}$. All stability studies were conducted using 50, 250, and $500 \mathrm{ng} / \mathrm{mL}$ of cIBR7 and ADTC5 with three determinations for each concentration of peptide.

\section{Statistical Analysis}

For brain delivery of peptides and galbumin, the differences in the brains treated with or without BBB modulators were compared using ANOVA with Student-Newman-Keuls for determining the statistical significance. A $p$-value of less than 0.05 was used as a criterion for statistical significance. 


\section{Results}

\section{In Vivo Brain Delivery of Galbumin in Balb/c Mice as Detected by MRI}

The activity of cadherin peptides (i.e., HAV6, HAV4, cHAVc3, and ADTC5) to enhance the brain delivery of galbumin into the brain of Balb/c mice was evaluated. The HAV6, cHAVc3, and ADTC5 treatment groups showed a significant increase in the brain deposition of galbumin in the posterior, midbrain, anterior regions compared to those of vehicle (Figures 1A-D). The enhanced brain delivery of galbumin was observed as early as $3 \mathrm{~min}$ after the i.v. injection (Figures 1B-D). In contrast, the HAV4 treatment group did not show an enhancement of galbumin brain deposition. From the AUC (Figure 1D), the levels galbumin enhancement were in the following order: posterior $>$ midbrain $>$ anterior.

To evaluate the duration of BBB opening for large macromolecules, galbumin was delivered 10 or 40 min after administration of HAV6 and ADTC5 peptides in a pretreatment experiment. Time-dependent galbumin brain depositions are shown in Figures 2A-D for 10-min pretreatment and in Figures 2A and 2E-G for 40-min pretreatment. The galbumin AUCs were also shown on immediate as well as 10- and 40-min pretreatment experiments (Figure 3). As previously shown, immediate delivery of galbumin with the HAV6 or ADTC5 peptide produced significant enhancements of galbumin brain deposition in all brain regions. After 10-min pretreatment with ADTC5, that there was still significant enhancement of brain deposition of galbumin while no enhancement was observed with HAV6 pretreatment. Using 40-min pretreatment, there was no observed galbumin enhancement produced by ADTC5 or HAV6.

\section{In Vivo Brain Delivery of R800cw-cLABL in Balb/c Mice as Detected by NIRF Imaging}

The brain delivery of a 12-amino acid peptide, IRdye800cw-cLABL (MW = $2182.4 \mathrm{~g} / \mathrm{mol}$ ), was accomplished via i.v. administration in the presence and absence of ADTC5 (Figure 4). Twenty minutes after i.v. administration, the mice were sacrificed. NIRF imaging was used to detect peptide brain deposition. The labeled cLABL peptide delivered with ADTC5 has qualitatively higher brain fluorescence than that without ADTC5 (Figures 4A-B). Quantitatively, the brain-to-plasma ratio was used to determine the effect of ADTC5; the brain deposition of CLABL with ADTC5 was about 3.5-fold higher than that without ADTC5 (Figure 4C, bottom panel).

\section{LC-MS/MS Method Development for Quantification of clBR7 in Rat Brain}

For calculating the amounts of peptides in the rat brains, liquid chromatography and tandem mass spectroscopy conditions were optimized to achieve the best separation and quantification of cIBR7, ADTC5, and the IS peptides in the rat brain extracts. The standard solution for each peptide was infused into the mobile phase using a syringe; the dominant peaks were observed with mass-to-charge ratios of $\mathrm{m} / \mathrm{z}$ 776.5, 773.5, and 395.9 for cIBR7, ADTC5, and IS, respectively. MRM was used for each precursor ion to produce three respective transitions, including $\mathrm{m} / \mathrm{z}$ $776.5 \rightarrow \mathrm{m} / \mathrm{z} 70.2$ for cIBR7; m/z $773.5 \rightarrow \mathrm{m} / \mathrm{z} 294.1$ for ADTC5 and m/z $395.9 \rightarrow \mathrm{m} / \mathrm{z} 86$ for IS using optimized collision energies of $120 \mathrm{~V}, 35 \mathrm{~V}$, and $35 \mathrm{~V}$, respectively.

\section{Validation of Mass Spectrometric Method}

\section{Selectivity}

Selectivity of the method was evaluated by analyzing chromatograms of blank brain homogenate samples and blank brain homogenates spiked with cIBR7, ADTC5, and IS. The results showed no endogenous interferences at the retention times of the three analytes (Figure 5). The retention times for cIBR7, IS, and ADTC5 were $2.88 \mathrm{~min}, 3.80 \mathrm{~min}$, and $6.10 \mathrm{~min}$, respectively.

\section{Linearity, Accuracy, and Precision}

Calibration curves for cIBR7 and ADTC5 were prepared in concentrations ranging from 50 to 500 $\mathrm{ng} / \mathrm{mL}$ to determine the lowest limit of detection (LLOD) and intra-day and inter-day accuracy and precision (Table 3). In this case, the peak area ratios of cIBR7-to-IS and ADTC5-to-IS were plotted separately. Good linearity was observed for both cIBR7 and ADTC5 with $R^{2} \geq 0.99$. The LLOD for cIBR7 and ADTC5 were $15 \mathrm{ng} / \mathrm{mL}$ and $10 \mathrm{ng} / \mathrm{mL}$, respectively. Intra-day and inter-day accuracy and precision were determined by analyzing samples at three concentration levels for \%RSD and \%RE (Table 3). The $\%$ RSD and \%RE values were less than 15\% for intra-day and inter-day for both analytes.

Table 3. Precision and Accuracy for clBR7 and ADTC5 $(n=3)$

\begin{tabular}{llllll}
\hline Analytes & $\begin{array}{l}\text { Concentration } \\
(\mathrm{ng} / \mathrm{mL})\end{array}$ & \multicolumn{2}{c}{ Intra-day } & \multicolumn{3}{c}{ Inter-day } \\
\cline { 3 - 6 } & \%SD & \%RE & \%RSD & \% RE \\
\hline cIBR7 & 50 & 10.1 & 14.1 & 9.6 & 11.2 \\
& 250 & 9.9 & 4.1 & 9.1 & -1.9 \\
& 500 & 8.5 & 2.3 & 6.4 & 3.3 \\
ADTC5 & 50 & 7.4 & 6.6 & 12.4 & 3.7 \\
& 250 & 14.6 & -3.3 & 6.7 & 2.1 \\
& 500 & 10.5 & 2.5 & 7.1 & 4.2 \\
\hline
\end{tabular}


A

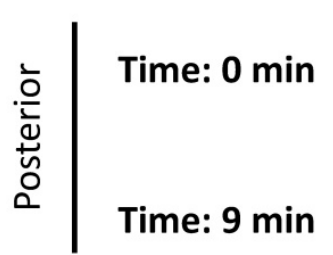

Control

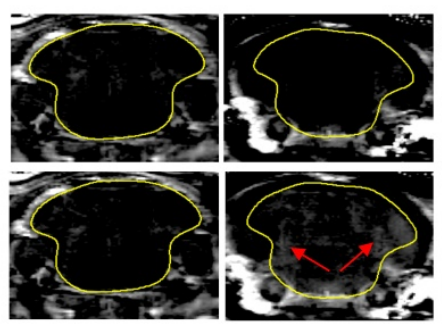

Control

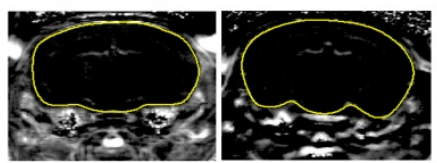

Time: $9 \mathrm{~min}$

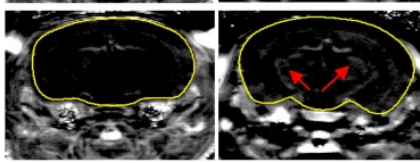

Control

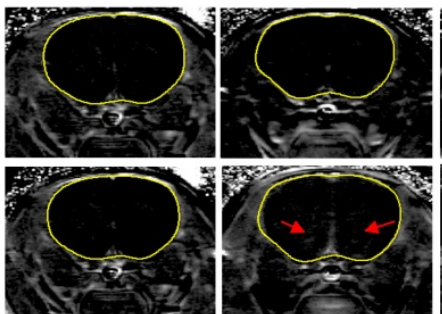

Control

Hav6 $10.010 \mathrm{mmol}$

Posterior

Time: $0 \mathrm{~min}$

Time: $9 \mathrm{~min}$

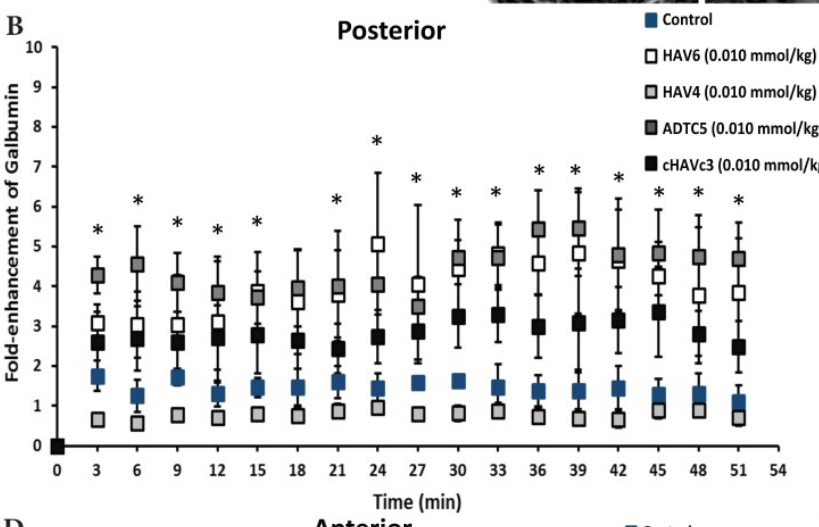

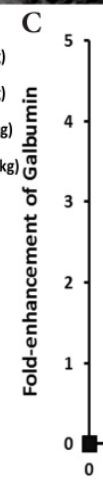

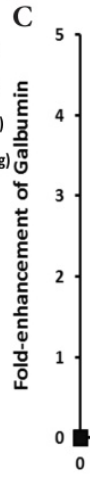

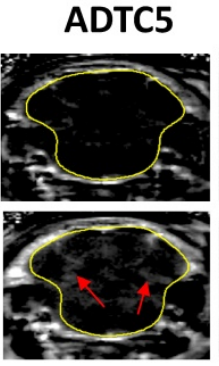

ADTC5
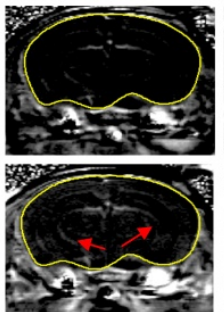

ADTC5

cHAVc3

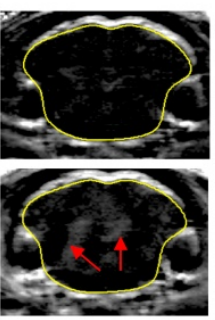

cHAVC3

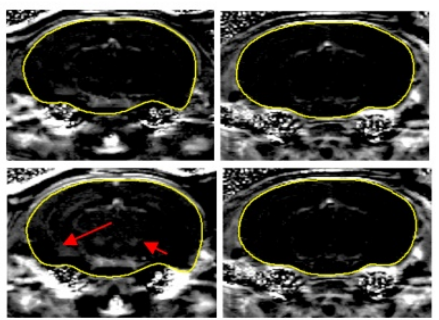

HAV4

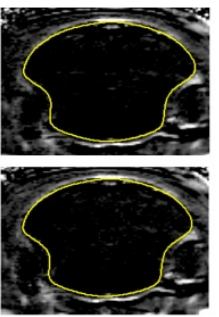

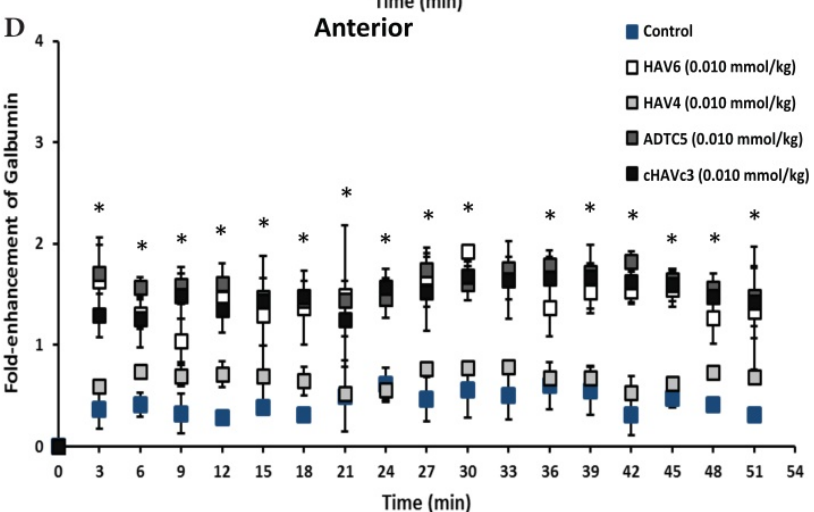

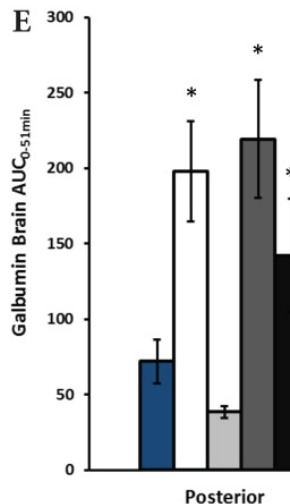

Time (min)

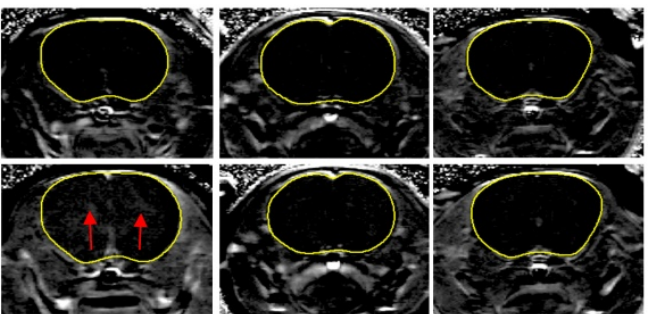

Mid Brain

Control

口 HAV6 $(0.010 \mathrm{mmol} / \mathrm{kg})$ 口 HAV4 $(0.010 \mathrm{mmol} / \mathrm{kg})$ $\begin{array}{cccccc} & * & * & * & \square \text { ADTC5 }(0.010 \mathrm{mmol} / \mathrm{kg}) \\ * & & * & *\end{array}$

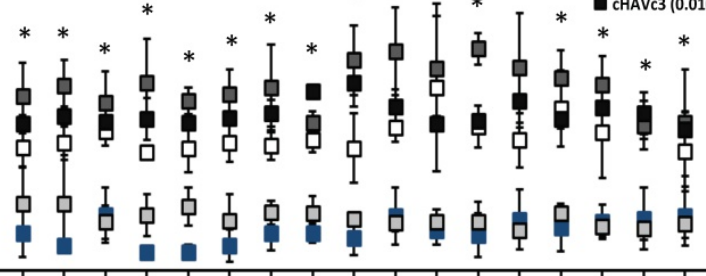

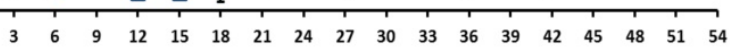

Control

口 HAV6 $(0.010 \mathrm{mmol} / \mathrm{kg})$

口 HAV4 $(0.010 \mathrm{mmol} / \mathrm{kg})$

$\square$ ADTC5 $(0.010 \mathrm{mmol} / \mathrm{kg})$

D cHAVc3 (0.010 mmol/kg)

Figure 1. The effects of cadherin peptides (HAV6, HAV4, ADTC5, and cHAVc3) on enhancing brain delivery of $65 \mathrm{kDa}$ galbumin as monitored MRI (A) T1-weighted $M R$ images of brain depositions of galbumin at 0 and 9 min time points when delivered with and without peptide. The depositions of galbumin were observed in the posterior, midbrain, and anterior regions as gray spots (see red arrows). (B-D) The time-dependent brain depositions of galbumin in the (B) posterior, (C) midbrain, and (D) anterior regions as monitored by MRI every 3 min over a 51-min imaging session. (E) The AUC of galbumin brain depositions when delivered with peptides or control vehicle. Star $\left(^{*}\right)$ represents statistical significant with $p<0.05$ as evaluated using ANOVA with Student-Newman-Keuls post hoc comparison of the means $(n=3)$. 
A

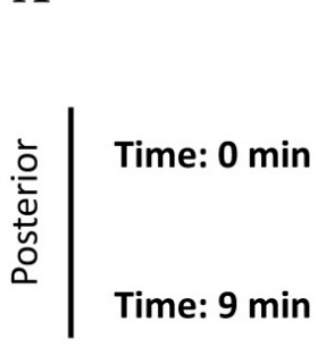

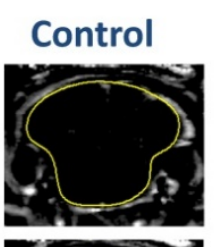

$10 \mathrm{~min}$ pretreatment

40 min pretreatment

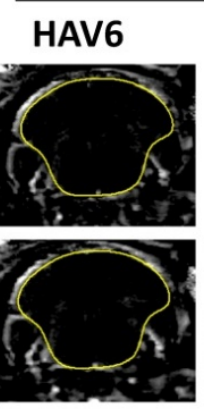

ADTC5

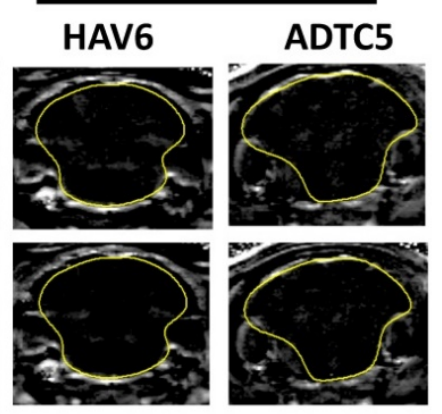

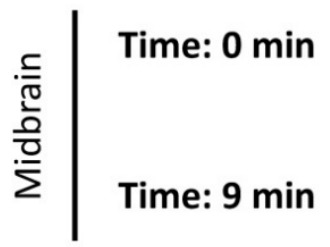
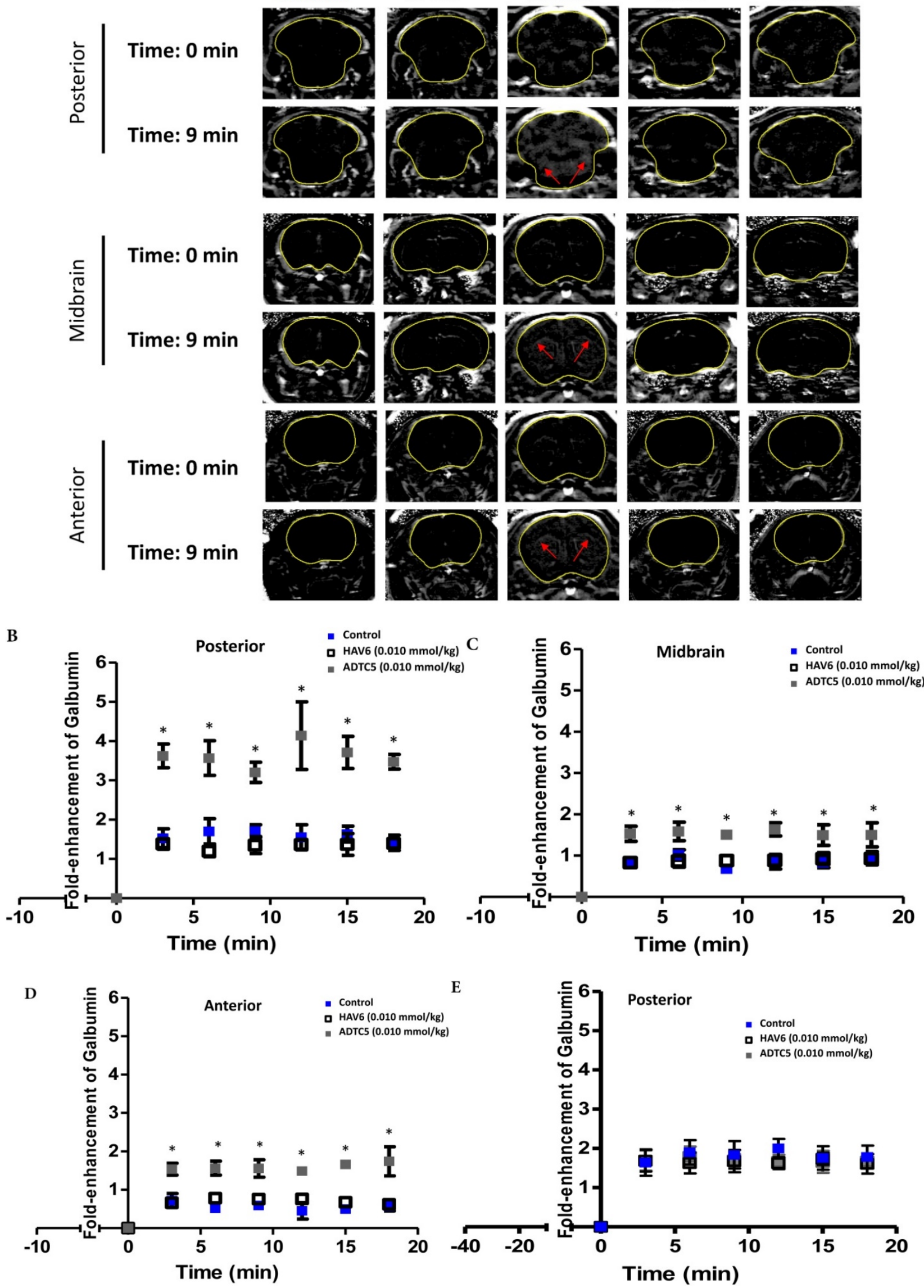

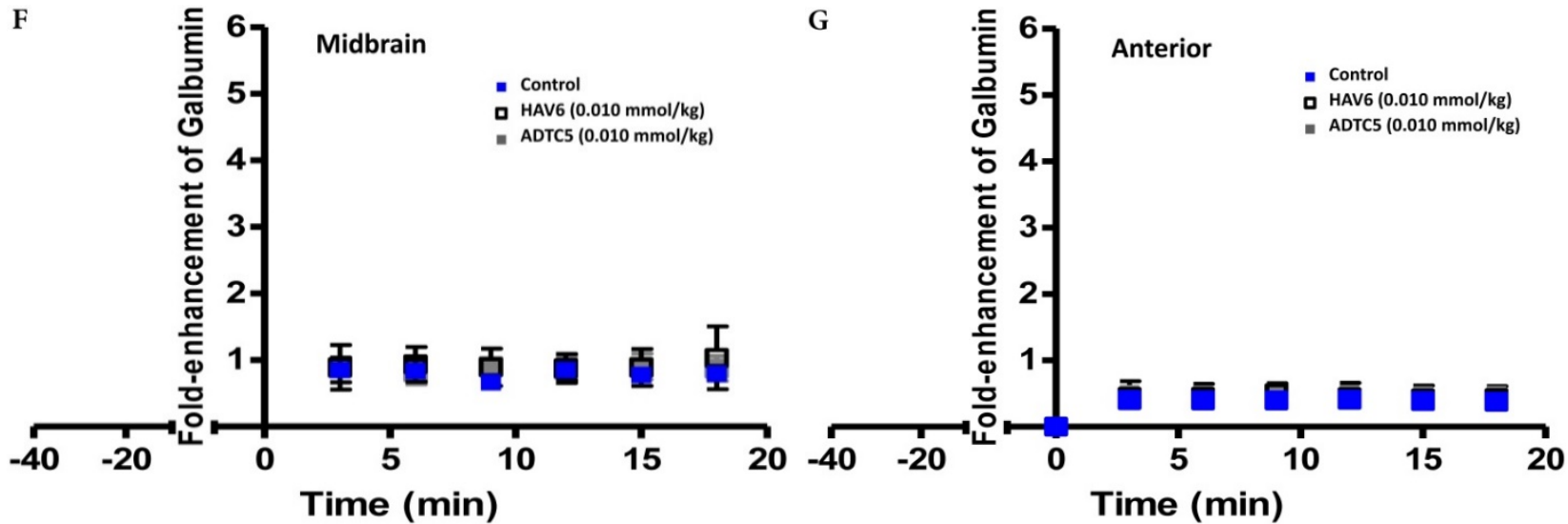

Figure 2. Comparison of 10-min and 40-min pretreatment with HAV6 or ADTC5 peptide on the brain depositions of galbumin. (A) T1-weighted MR image comparisons of brain depositions of galbumin after 10-min and 40-min pretreatment with HAV6 and ADTC5. The brain depositions of galbumin were observed as gray spots (see red arrows) in the posterior, midbrain, and anterior regions at 0 and 9 min time points using MRI. (B-D) Time-dependent brain depositions of galbumin observed at the (B) posterior, (C) midbrain, and (D) anterior regions when delivered 10 min after HAV6 or ADTC5 administration. (E-G) Time-dependent brain depositions of galbumin observed at the (E) posterior, (F) midbrain, and (G) anterior regions when delivered 40 min after HAV6 or ADTC5 administration. Star $\left(^{*}\right)$ represents statistical significance with $p<0.05$ as evaluated using ANOVA with Student-Newman-Keuls post hoc comparison of the means $(\mathrm{n}=3)$.
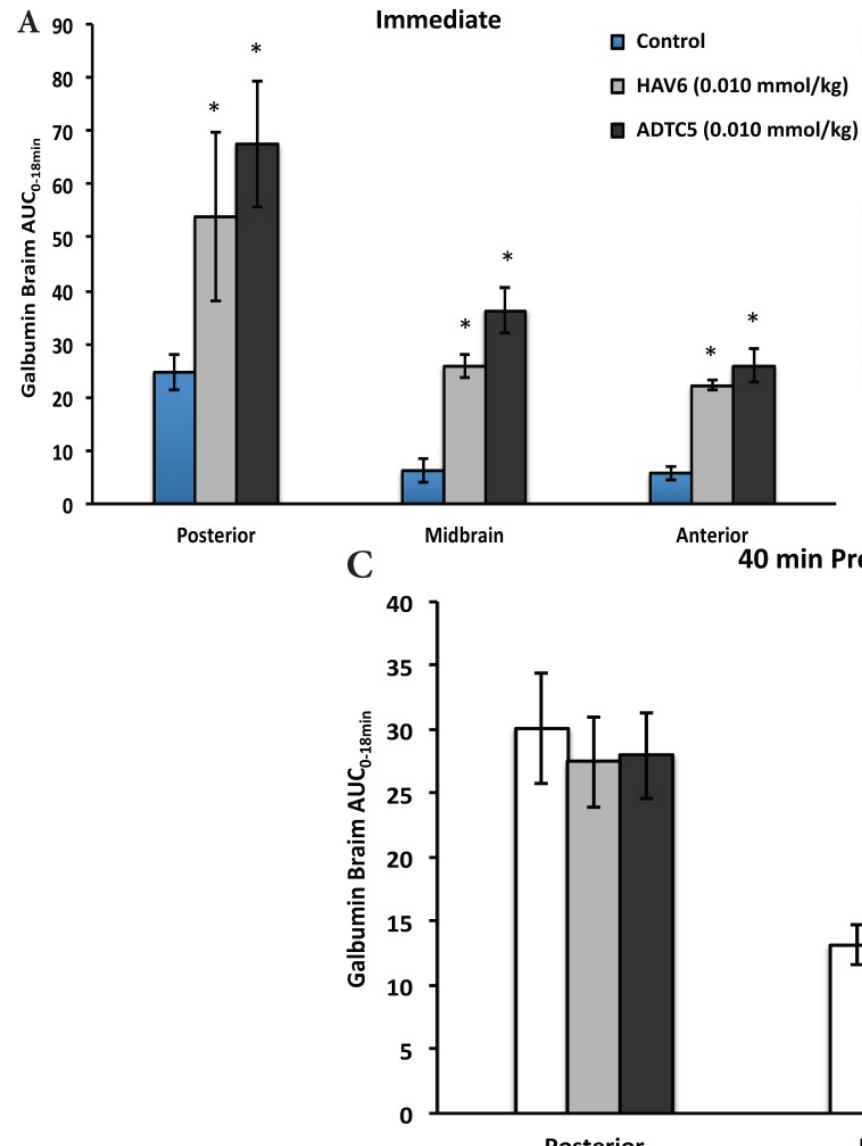

Posterior
B

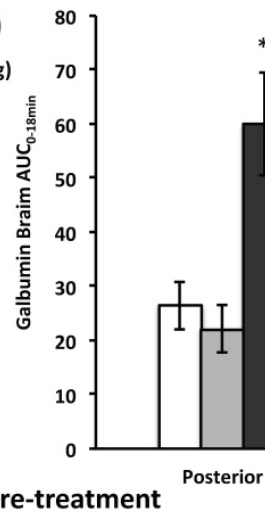

10 min Pre-treatment

口 Control

口 HAV6 $(0.010 \mathrm{mmol} / \mathrm{kg})$

ADTC5 $(0.010 \mathrm{mmol} / \mathrm{kg})$ 든ol

口 HAV6 (0.010 mmol/kg)

口 ADTC5 $(0.010 \mathrm{mmol} / \mathrm{kg}$ )
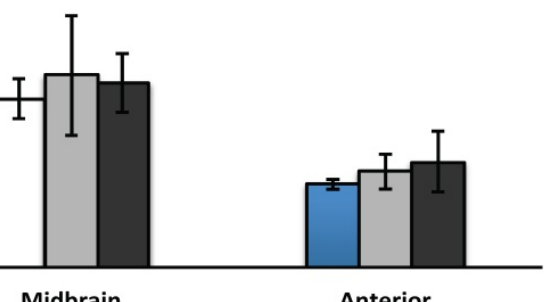

Figure 3. Comparisons of the AUC of galbumin brain deposition upon (A) immediate, (B) 10-min, and (C) 40-min pretreatment with HAV6 and ADTC5 peptides. (A) Immediate delivery with HAV6 or ADTC5 peptide significantly enhanced brain deposition of galbumin in posterior, midbrain, and anterior regions. (B) With 10-min pretreatment, ADTC5 still significantly enhanced the delivery of galbumin while HAV6 did not. (C) With 40-min pretreatment, there was no enhancement in galbumin accumulation in the brain with either ADTC5 or HAV6 peptide. Star $(*)$ represents statistical significant with $p<0.05$ as evaluated using ANOVA with Student-Newman-Keuls post hoc comparison of the means $(n=3)$. 

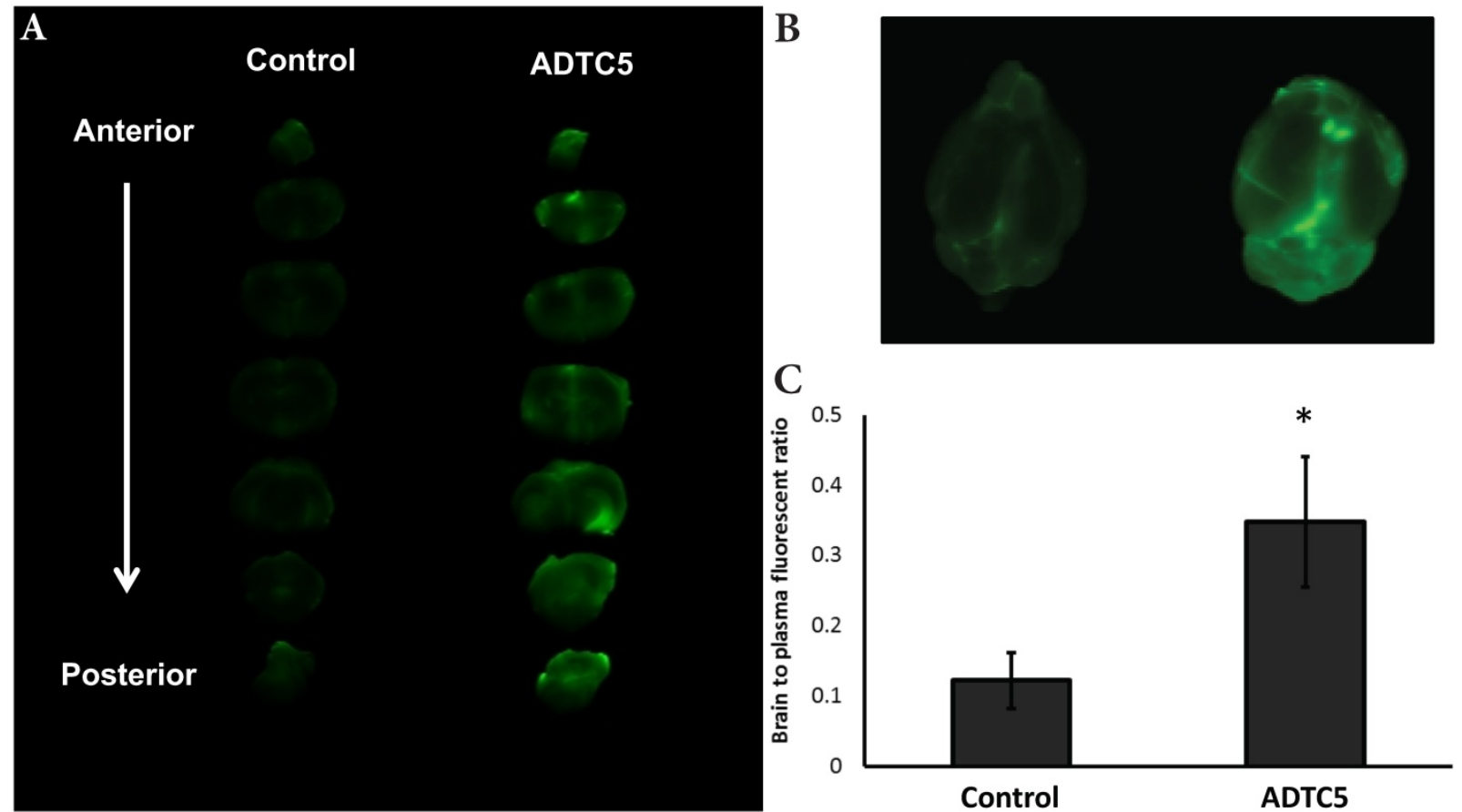

Figure 4. The effect of ADTC5 peptide on increasing the brain deposition of IRdye $800 \mathrm{cw}$-cLABL peptide after i.v. administration in Balb/c mice as detected by NIRF imaging. (A) The images of brain depositions of IRdye $800 \mathrm{cw}$-cLABL in different slices of the brain from anterior to posterior regions when delivered with vehicle (left) and ADTC5 (right). (B) The additive representation of brain depositions of IRdye $800 \mathrm{cw}$-cLABL when delivered with vehicle (left) or ADTC5 (right). (C) Comparison of brain-to-plasma ratios of IRdye $800 \mathrm{cw}-\mathrm{cLABL}$ peptide when administered with vehicle (control) and ADTC5. Star $(*)$ indicates statistical significance with $p<0.05$; ADTC5-treated group, $n=4$; control group, $n=3$. The error bars represent SD.
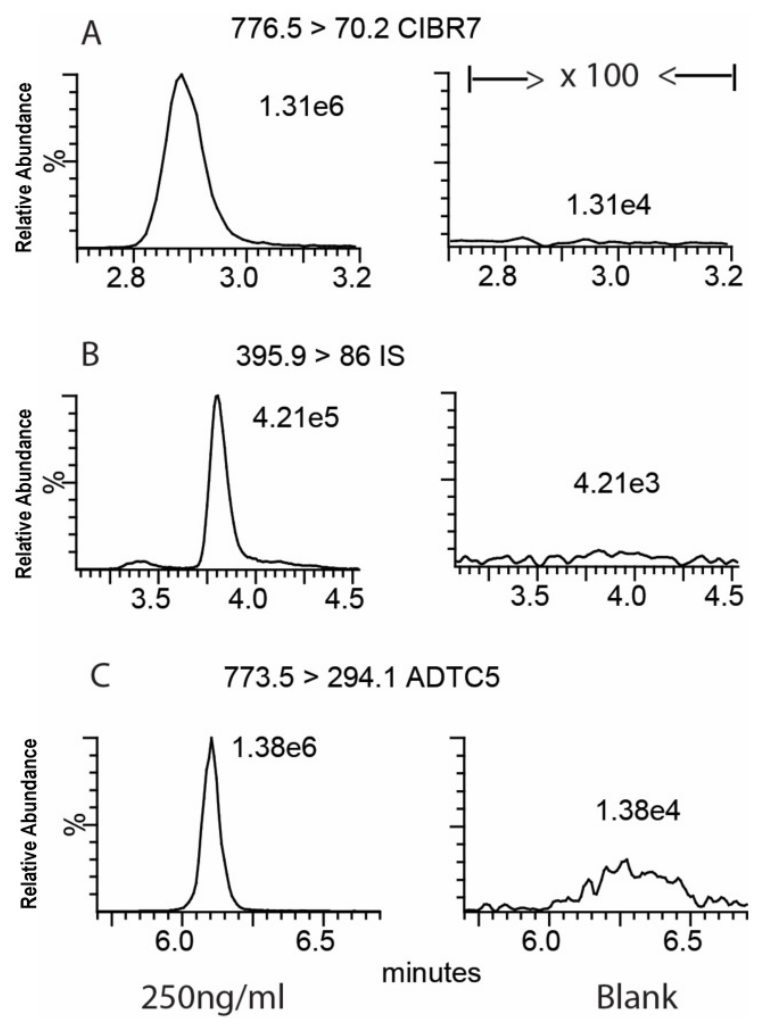

Figure 5. MRM chromatograms of brain extracts with (A) clBR7 peptide (left) and blank (right), (B) IS peptide (left) and blank (right), and (C) ADTC5 peptide (left) and blank (right). The peptide separation was done using a Luna UPLC $C_{18}$ column with $2.1 \mathrm{~mm} \times 50 \mathrm{~mm}$ dimensions, particle size $5 \mu \mathrm{m}$, and pore size 100 $\AA$ (Phenomenex, Inc., Torrance, CA). The elution was done using a binary gradient mobile phase consisting of solvent $\mathrm{A}: \mathrm{H}_{2} \mathrm{O}$ :formic acid (99.92:0.08) and solvent B: acetonitrile:formic acid (99.92:0.08).

\section{Stability and Extraction Recovery}

The potential instability of cIBR7 and ADTC5 during the experiments was determined in three different peptide concentrations, temperatures, and storage conditions (Table 4). The variability of each peptide in the brain extract was calculated to be less than 15\% (\%RSD) (Table 4). The extraction recoveries of cIBR7 and ADTC5 from the brain homogenates were calculated by comparing the extracted and spiked samples. At three concentrations, the calculated mean recoveries for cIBR7 and ADTC5 were $90 \%$ and $92 \%$, respectively (Table 5).

Table 4. Stability of clBR7 and ADTC5 in brain extract $(n=3)$

\begin{tabular}{|c|c|c|c|c|}
\hline \multirow[t]{2}{*}{ Analyte } & \multirow{2}{*}{$\begin{array}{l}\text { Concentration } \\
(\mathrm{ng} / \mathrm{mL})\end{array}$} & \multicolumn{3}{|l|}{$\%$ RSD } \\
\hline & & $\begin{array}{l}\text { Room } \\
\text { Temp. }\end{array}$ & $-20^{\circ} \mathrm{C}$ & $\begin{array}{l}\text { Three freeze } \\
\text { thaw cycles }\end{array}$ \\
\hline \multirow[t]{3}{*}{ cIBR7 } & 50 & 7.79 & 13.54 & 9.72 \\
\hline & 250 & 9.48 & 1.90 & 5.72 \\
\hline & 500 & 8.73 & 8.96 & 6.34 \\
\hline \multirow[t]{3}{*}{ ADTC5 } & 50 & 12.18 & 9.18 & 13.12 \\
\hline & 250 & 10.61 & 0.45 & 11.01 \\
\hline & 500 & 6.95 & 7.16 & 8.97 \\
\hline
\end{tabular}

\section{In Vivo Brain Delivery of cIBR7 in Sprague-Dawley Rats}

The brain delivery of cIBR7 was evaluated in Sprague-Dawley rats following bolus i.v. injection of 
cIBR7 either alone or in combination with the cadherin peptide ADTC5. The amount of cIBR7 in the brain in the presence of ADTC5 was significantly higher (approximately four times) than that without ADTC5 $(p=0.013$, Figure 6$)$. In the presence of ADTC5, the average total amount of cIBR7 was $855 \pm$ $171 \mathrm{ng} / \mathrm{g}$ brain while it was $213 \pm 53 \mathrm{ng} / \mathrm{g}$ brain in the absence of ADTC5. Brain accumulation of cIBR7 was also determined in brain homogenates following capillary depletion to remove any cIBR7 within the brain vasculature. In this case, the ratios of cIBR7-to-IS without and with vasculature depletion were 0.133 and 0.128 , respectively. This suggests that there is no difference between the two methods.

Table 5. Extraction recovery of cIBR7 and ADTC5 from rat brain homogenate

\begin{tabular}{lll}
\hline Analytes & Concentration $(\mathrm{ng} / \mathrm{mL})$ & Extraction Recovery $(\%)$ \\
\hline cIBR7 & 50 & 89 \\
& 250 & 90 \\
& 500 & 92 \\
ADTC5 & 50 & 90 \\
& 250 & 92 \\
& 500 & 94 \\
\hline
\end{tabular}

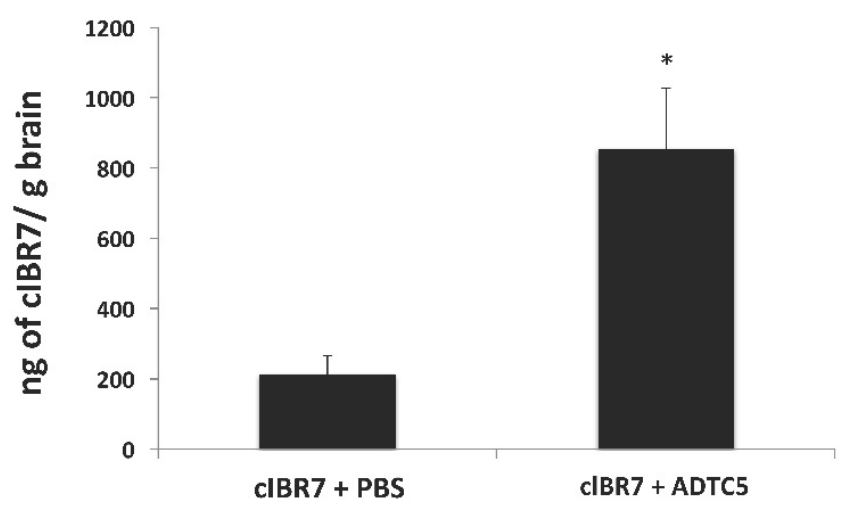

Figure 6. The effect of ADTC5 peptide on enhancing brain delivery of clBR7 peptide in rats as detected by LC-MS/MS. cIBR7 $(40 \mathrm{mg} / \mathrm{kg})$ was delivered in the presence or absence of ADTC5 $(30 \mathrm{mg} / \mathrm{kg})$. The rats were sacrificed after 10 minutes, and the whole brains were collected for analysis. The total amount of clBR7 in rat brains after in vivo delivery via i.v. administration was significantly higher in the presence of ADTC5 compared to control with PBS treatment. Star $(*)$ indicates a significant difference from the control study with $p<0.05$. Error bars show the mean \pm SE from ADTC5-treated group $(n=5)$ and PBS-treated group $(n=4)$.

\section{Discussion}

The delivery of peptides and proteins including antibodies (MW 150 kDa) or Fab-fragment of antibodies (MW 50 kDa) into the brain for diagnosis and treatment of brain diseases had been a topic of great interest. However, success in delivering them to the brain has been very limited because they have physicochemical properties (e.g., size, hydrogen-bonding potential, and conformation) that prevent them from partitioning into cell membranes to cross the BBB [1]. There is clearly a need to develop methods to deliver peptides and proteins into the brain. The ability to deliver functional peptides and proteins, including antibodies, into the brains of normal and diseased animals (i.e., mice and rats) would provide advantages in treating various brain pathologies and studying brain functions at the cellular level. Previously, cadherin peptides have been shown to improve in vivo delivery of small-to-large marker molecules, including $25 \mathrm{kDa}$ IRdye-800cw PEG, into the brains of mice and/or rats $[16-18,20]$. In in vivo Balb/c mice, HAV6 peptide also enhances the delivery of IRdye-800cw PEG in the lung, kidney, and small intestine [17]. It should be noted that the PEG molecule has physicochemical properties different from those of peptides and proteins in that they have a variety of functional groups as well as secondary or tertiary structures.

The first aim was to determine brain delivery of a large protein, $65 \mathrm{kDa}$ galbumin, using several known cadherin peptides (i.e., HAV6, HAV4, cHAVc3, or ADTC5) in Balb/c mice. Galbumin is a conjugate between albumin and Gd-complex; thus, it can be detected and quantified in the brains of living animals using MRI. Compared to vehicle control, ADTC5, HAV6, and cHAVc3 peptides significantly enhanced the delivery of galbumin into all three regions of the brain (i.e., posterior, midbrain, and anterior). The enhancement was immediate - within 3 min after administration of the modulator peptide with galbumin (Figure 1B-D). Our hypothesis is that cadherin peptides open the intercellular junctions to create small, medium, and large pores in the paracellular pathways of the BBB as soon as the peptide is injected [18]. It is interesting to find that HAV4 did not enhance galbumin brain delivery (Figure 1D); however, HAV4 did enhance brain delivery of Gd-DTPA [20]. This suggests that HAV4 creates small pores but is unable to create the large pores in the intercellular junctions of the BBB required for delivery of macromolecules to the brain. Overall, ADTC5, HAV6, and cHAVc3 can effectively enhance the brain delivery of a protein as large as $65 \mathrm{kDa}$ in Balb/c mice.

The duration of the BBB opening created by HAV6 and ADTC5 peptides to allow large molecules to enter the brain is an important parameter for avoiding unwanted side effects of this method. The opening of BBB paracellular pathways for brain delivery should be tightly controlled to avoid BBB permeation of unwanted large molecules and immune cells (e.g., macrophages, $\mathrm{T}$ cells, and B cells) entering the brain. Therefore, the time-dependent 
effect of pretreatment using HAV6 or ADTC5 on the $\mathrm{BBB}$ permeation of galbumin was investigated. As expected, immediate treatment with HAV6 or ADTC5 peptide along with galbumin showed enhanced brain deposition of galbumin (Figure 3A). In contrast, ADTC 5 but not HAV6 enhanced the brain delivery of galbumin when a 10-min peptide pretreatment experiment was conducted (Figure 3B). This indicates that the duration of the $\mathrm{BBB}$ opening provided by HAV6 peptide for a large molecule is less than $10 \mathrm{~min}$ and shorter than that produced by ADTC5. In contrast, the BBB opening for a large molecule by ADTC5 lasts longer than $10 \mathrm{~min}$. After 40-min pretreatment, there was no enhancement of galbumin deposition in the brain by either peptide. This suggests that the duration of BBB opening for ADTC5 was longer than $10 \mathrm{~min}$ but shorter than $40 \mathrm{~min}$. Overall, for both HAV6 and ADTC5, the BBB opening for large molecular weight molecules was reversible and short.

In previous studies, HAV6, HAV4, $\mathrm{cHAVc} 3$, and ADTC5 peptides significantly enhanced the brain delivery of a small molecular weight vasculature marker, Gd-DTPA (MW $=547.575 \mathrm{~g} / \mathrm{mol})$, in a dose-dependent manner $[17,18,20]$. To determine the duration of BBB opening created by cadherin peptides for a small molecule, pretreatment studies were carried out using Gd-DTPA and detected by MRI. Following a 1-h pretreatment with peptide, linear HAV6 and HAV4 peptides did not aid the penetration of Gd-DTPA through the BBB, suggesting that the $\mathrm{BBB}$ opening created by these linear peptides lasts less than $1 \mathrm{~h}$. In contrast, cyclic ADTC5 and cHAVc3 peptides create a BBB opening for Gd-DTPA delivery of more than $2 \mathrm{~h}$ but less than $4 \mathrm{~h}[18,20]$. Therefore, cyclic ADTC5 and cHAVc3 peptides created a longer lasting BBB opening than did linear HAV6 and HAV4 peptides. The data suggest that both HAV6 and ADTC5 peptides create a longer duration of BBB opening for a small molecule (i.e., Gd-DTPA) than a large molecule (i.e., galbumin). To explain the observations in the pretreatment studies, it is proposed that cadherin peptides modulate the BBB by immediately creating small, medium, and large pores in the intercellular junctions. However, the large pores quickly collapse to medium and small pores and, finally, the medium pores collapse to small pores in the intercellular junctions of the BBB.

To evaluate brain delivery of peptides, a 12-amino acid residue peptide, IRdye800cw-cLABL peptide, was delivered with ADTC5 in Balb/c mice. The peptide brain deposition was detected using NIRF imaging, which has an advantage in its ease of quantifying the molecule. The administration of ADTC5 peptide significantly enhanced brain delivery of cLABL peptide compared to control. The data suggest that the mechanism of transport of cLABL peptide was via passive diffusion through the paracellular pathway of the BBB. Although cLABL peptide could bind to and be internalized by ICAM-1 on the surface of BBB endothelial microvessels, the brain deposition of this peptide when delivered with vehicle was very low, suggesting that ICAM-1 receptor-mediated endocytosis was not involved (Figure 4). cLABL peptide was selected because it binds to ICAM-1 protein on the surface of epithelial, endothelial, and immune cells. ICAM-1 is upregulated during inflammation [22, 23]; thus, it can be used as a target protein for labeled cLABL to detect upregulation of ICAM-1 during neuroinflammation. cLABL peptide is derived from the binding region of the I-domain of LFA-1 to the domain-1 (D1) of ICAM-1 [28]. It binds to ICAM-1 and inhibits LFA-1/ICAM-1-mediated T-cell adhesion to epithelial cells [29]. In addition, it is also internalized by ICAM-1 in activated Molt-3 T cells [23].

The final aim is to develop a method to detect and quantify an intact peptide in the brain without the use of any radioactive, MRI, or NIRF label. cIBR7 peptide was selected for brain delivery using ADTC5 peptide in Sprague-Dawley rats; ADTC5 has not been evaluated in modulating the BBB of in rats in vivo. Previously, ADTC5 peptide was shown to enhance brain delivery of ${ }^{14} \mathrm{C}$-mannitol in an in situ rat brain perfusion model [18]. In addition, HAV6 peptide has been shown to enhance brain delivery of an MRI contrast agent, Gd-DTPA, in in situ brain perfusion and in vivo in Sprague-Dawley rats [16]. These results suggest that ADTC5 could also enhance the brain delivery of cIBR in rats. To detect the brain deposition of cIBR7, an extraction as well as a sensitive and selective LC-MS/MS method were developed to detect and quantify the delivered peptide in the rat brain. cIBR7 peptide was delivered in the presence of ADTC5 or control by injection via the jugular vein. After $10 \mathrm{~min}$, the rat was sacrificed and the BBB was perfused with saline to remove potential residual peptides in the vascular endothelial cells to ensure accurate measurement of the analyte concentration in the brain. ADTC5 significantly enhanced the brain delivery of unlabeled cIBR7 peptide by as much as 4 -fold (Figure 6, $p=0.013$ ), and $855 \pm 171$ or $213 \pm 53$ $\mathrm{ng} / \mathrm{g}$ brain of cIBR7 was detected when delivered using ADTC5 or vehicle, respectively. In summary, this study showed that ADTC5 enhanced the brain delivery of intact cIBR7 in Sprague-Dawley rats, and that the extraction and LC-MS/MS detection methods were sensitive to quantified cIBR7 brain deposition.

cIBR7 peptide was selected because it binds to LFA-1 receptors on the surface of $\mathrm{T}$ cells and inhibits 
T-cell adhesion to Caco-2 cell monolayers [25]. cIBR7 is a smaller analog of the parent cIBR peptide, which is derived from the D1 of ICAM-1. cIBR peptide has been shown to bind to the I-domain of LFA-1 receptor $[25,26,30]$. cIBR7 and cIBR peptides can be internalized by LFA-1 into the cytoplasmic domain of immune cells (i.e., T cells, H-60 leukemic cells) [24, 31]; therefore, labeled cIBR and cIBR7 peptides have the potential to detect LFA-1-expressing immune cells in the brain.

It is interesting to find that the ADTC5 peptide was also detected in a lower amount (about $23 \mathrm{ng} / \mathrm{g}$ brain) than cIBR7 (855 ng/g brain) in the brain although they were administered at almost similar dosage levels (i.e., $40 \mathrm{mg} / \mathrm{kg}$ cIBR7 and $30 \mathrm{mg} / \mathrm{kg}$ ADTC5). There are several potential reasons for this difference. First, ADTC5 could bind to cadherins in the adherens junctions of the BBB to lower the amount of free ADTC5 that would be available to penetrate the BBB. The half-lives $\left(t_{1 / 2}\right)$ of plasma stabilities of ADTC5 and cIBR7 were $5.7 \mathrm{~h}$ and $2.8 \mathrm{~h}$, respectively, suggesting that the low amount of ADTC5 in the brain was not due to its instability in plasma. Another alternative explanation is that CIBR7 and ADTC5 have vastly different pharmacokinetic and pharmacodynamic profiles; thus, further studies are needed to understand the difference in their brain depositions.

This study developed and validated an extraction as well as a sensitive LC-MS/MS method to quantify the intact cIBR7, ADTC5, and IS in rat brains after in vivo studies. The results showed that cIBR7 peptide could be detected confidently as an intact molecule in the brain. The optimized extraction was very efficient, and it recovered higher than $90 \%$ of the intact cIBR7 and ADTC5 peptides from the brain (Table 5). The recoveries of cIBR7 and ADTC5 peptides from the brain were accomplished by optimizing the homogenization medium, composition of extraction solvent, and the reconstitution solvent mixture. In addition, this study also confirmed our previous observation on brain delivery of labeled molecules (i.e., radioactive, IRdye, and Gd-DTPA). It also suggests that the detected labeled molecules could reasonably represent the intact molecules in the brain and are not due to detection of the label or molecular fragments of the parent molecules.

IS peptide was designed as a reference molecule for the LC-MS/MS analysis to maximize the precision of peptide quantification in the brain and it has similar physicochemical properties as CIBR7 peptide. It was also used to account for errors and losses during sample handling and ionization during LC-MS/MS experiments. IS peptide was designed to have physicochemical properties similar to those of
cIBR7 peptide. In this case, the Val6 residue in cIBR7 peptide was replaced with the Ile6 residue, and this simple change in IS provided a longer retention time (3.8 $\mathrm{min}$ ) for IS compared to cIBR7 peptide $(2.88 \mathrm{~min}$ ) in the C18 column of LC-MS/MS (Figure 5). The ADTC5 peptide was eluted at $6.10 \mathrm{~min}$, which is 3.8 min later than IS peptide; therefore, all three peptides were well separated in the column. All three peptides (cIBR7, IS, ADTC5) were readily water soluble and, thus, no other additives or co-solvents were need for peptide reconstitution during the study.

To improve the sensitivity of analyte detection, mass spectrometry (MS) parameters were initially optimized using a direct infusion of each analyte to the instrument, and the MRM of several stable transitions were optimized for each analyte. The best transition with the highest intensity was selected for each analyte. Then, the method validations for QC samples were carried out to determine selectivity, linearity, accuracy, precision, sensitivity, and stability over a range of concentrations in the calibration curve at $50-500 \mathrm{ng} / \mathrm{mL}$ following FDA guidelines [32]. The results showed that the LC-MS/MS method was selective for all three analytes with no interferences. A good linearity was achieved over a concentration range of $50-500 \mathrm{ng} / \mathrm{mL}$ for cIBR7 and ADTC5. Intra-day and inter-day precision and accuracy variability for both cIBR7 and ADTC5 were less than 15\% (R.S.D and R.E) (Table 3), suggesting that the MS method is accurate and precise. Variability (\%R.S.D) for stability for both peptides was less than 15\%, suggesting that the analytes are stable in brain extract under different conditions. Thus, the developed LC-MS/MS method was very sensitive and reliable for detecting the amount of delivered peptides in the brain.

The hypothesis for the mechanism of action of cadherin peptides is that they bind in equilibrium fashion to the extracellular domain of E-cadherins to inhibit E-cadherin homophilic physical interactions (i.e., trans and/or cis interactions) among cadherins in the intercellular junctions in an equilibrium fashion [33]. The physical nature of this inhibition is not expected to change the expression of E-cadherin on the cell surface. Incubation of MDCK cell monolayers $(2-4$ h) with HAV6 peptide increased the total expression of E-cadherin in response to peptide modulation of the intercellular junction [14]. However, it is still not clear whether the same phenomenon occurs in the BBB. Although E-cadherin is found on the epithelial cells and VE-cadherin (cadherin-5) is found in the peripheral vascular endothelium [34], we found that proteins from bovine brain microvessel endothelial cells (BBMEC) react only with anti-E-cadherin antibody and not with 
anti-cadherin-5 and anti-N-cadherin [35]. Abbruscato and Davis indicated that BBMEC monolayers also expressed E-cadherin [36]. Vorbrodt et al. showed VE-cadherin in the rat endothelial BBB and a weak reaction with anti-E-cadherin [37]; however, Vorbort et al. [38] and Rubin et al. [34] suggested that VE-cadherin might not be associated with BBB function. Fluorescence-labeled HAV decapeptide and anti-E-cadherin $\mathrm{mAb}$ can bind to the intercellular juctions of the BBMEC monolayers as punctate structures as observed by fluorescence microscopy [35]. This labeled HAV peptide can also bind to single cells of BBMEC as detected by flow cytometry [35]. $\mathrm{HAV}$ peptides and anti-E-cadherin $\mathrm{mAb}$ also inhibit cadherin-mediated homotypic single cell aggregation of BBMEC [39]. Taken together, these results suggest that there is a potential role of E-cadherin in the BBB. At this point, it is not clear whether these peptides also bind to VE-cadherin.

To test whether cadherin peptides can bind to E-cadherin, the ${ }^{15} \mathrm{~N}$-labeled extracellular-1 (EC1) domain of E-cadherin was expressed and was titrated with cadherin peptides (i.e., ADTC5, HAV6, and cHAVc3). These cadherin peptides caused changes in chemical shifts of several amino acids on the EC1 as observed using heteronuclear single quantum correlation (HSQC) NMR spectroscopy experiments [33]. A combination of NMR spectroscopy and molecular docking experiments indicated that HAV6 and $\mathrm{CHAV} 3$ peptides have a different binding site than that of ADTC5 peptide. The binding site of HAV6 and cHAVc3 is at residues Y36, I38, F77, S78, and 194 on the EC1 domain [33]; in contrast, ADTC5 binds to residues I4, P5, P6, S8, and P10 on the $\mathrm{N}$-terminal $\beta$ sheet of the EC1 domain. To put these results into perspective, the $\mathrm{X}$-ray structure of C-cadherin was used to explain the potential mechanism of action of HAV- or ADT-peptides. $\mathrm{C}$-cadherin could form cis- and trans-interactions. The cis-interactions are formed via the EC1 domain of one molecule to the EC2 domain of a neighboring molecule on the same cell membranes [40]. In contrast, the trans-interactions are formed by domain swapping of two N-termini of one EC1 domain from two opposing cell membranes. In the trans-interaction, the Trp residue at the $\mathrm{N}$-terminus of one EC1 binds to a hydrophobic binding pocket of another EC1 domain from the opposing cell [40]. Using a combination of the X-ray structure of C-cadherin and our NMR binding and molecular docking studies, we hypothesize that HAV peptides block the EC1-EC2 cis-interactions of two cadherins from the same cell membranes to create greater porosity of the intercellular junctions of the BBB [33]. In contrast, ADTC5 binds to the domain-swapping region of two
EC1 domains for trans-interactions of E-cadherin from the opposing cell membranes. In summary, the results suggest that HAV6 peptide disrupts the intercellular junctions of the BBB via inhibition of cis-cadherin interactions while ADTC5 modulates the BBB opening by inhibiting the trans-cadherin interactions.

\section{Conclusion}

This study has shown that cadherin peptides enhance the delivery of functional molecules such as a $65 \mathrm{kDa}$ galbumin and peptides (i.e., cIBR7, cLABL). Both ADTC5 and HAV6 peptides create a short opening of the BBB for delivering a large molecule (i.e., galbumin) compared to a small molecule (e.g., Gd-DTPA). The intact cIBR7 peptide can be efficiently detected and quantified in the brain using LC-MS/MS. ADTC5 has been shown to modulate the BBB and enhance the brain delivery of molecules in mouse and rat models. The results suggest that there is compatibility of quantifying molecules in the brain using MRI, NIRF, and LC-MS/MS. Finally, BBB modulation by cadherin peptides depends on (a) the type and dose of modulator peptide, (b) the timing of delivery between BBB modulator and the delivered molecule, and (c) the size of the delivered molecules. These results further support potential uses of cadherin peptides in delivering molecules to the brains of animal models of brain diseases for treatment, diagnosis, and evaluation of mechanisms of progression of brain diseases.

\section{Acknowledgments}

Funding for this research was provided by an R01-NS075374 grant from the National Institute of Neurological Disorders and Stroke (NINDS), National Institutes of Health (NIH). We would like to thank Nancy Harmony for proofreading this manuscript.

\section{Abbreviations}

ANOVA: analysis of variance; BBB: blood brain barrier; CID: collision-induced dissociation; EC1: extracellular domain-1; ESI: electrospray ionization; FDA: Food and Drug Administration; Gd: DTPA: gadolinium-diethylenetriaminepentacetate; HPLC: high performance liquid chromatography; HSQC: heteronuclear single quantum correlation; IACUC: Institutional Animal Care and Use Committee; ICAM1: intercellular adhesion molecule-1; IRdye: infrared fluorescent dye; IS: internal standard; LFA-1: lymphocyte function-associated antigen-1; LOD: limit of detection; LOQ: limit of quantification; MRI: magnetic resonance imaging; MRM: multiple reaction monitoring; MS: mass spectrometry; NIH: National Institutes of Health; NINDS: National Institute of Neurological Disorders and Stroke; NIRF: 
near-infrared fluorescence; NMR: nuclear magnetic resonance; PEG: polyethylene glycols; QC: quality control; RE: relative error; ROI: regions of interest; RSD: relative standard deviation; TFA: trifluoroacetic acid.

\section{Competing Interests}

The authors have declared that no competing interest exists.

\section{References}

[1] Laksitorini M, Prasasty VD, Kiptoo PK, Siahaan TJ. Pathways and progress in improving drug delivery through the intestinal mucosa and blood-brain barriers. Ther Deliv. 2014; 5: 1143-1163.

[2] Pardridge WM. Blood-brain barrier delivery. Drug Discovery Today. 2007; 12: 54-61.

[3] Shannon RJ, Carpenter KLH, Guilfoyle MR, Helmy A, Hutchinson PJ. Cerebral microdialysis in clinical studies of drugs: pharmacokinetic applications. Journal of Pharmacokin Pharmacodyn. 2013; 40: 343-358.

[4] Grathwohl SA, Jucker M. Replacement of osmotic minipumps to extend the intracerebral infusion time of compounds into the mouse brain. Biotechniques. 2013; 55: 75-78.

[5] Huang Z, Cheng C, Jiang L, Yu Z, Cao F, Zhong J, Guo Z, Sun X. Intraventricular apolipoprotein ApoJ infusion acts protectively in Traumatic Brain Injury. J Neurochem. 2016; 136: 1017-1025.

[6] Chen Y, Liu L. Modern methods for delivery of drugs across the blood-brain barrier. Adv Drug Deliv Rev. 2012; 64: 640-665.

[7] Oberoi RK, Parrish KE, Sio TT, Mittapalli RK, Elmquist WF, Sarkaria JN. Strategies to improve delivery of anticancer drugs across the blood-brain barrier to treat glioblastoma. Neuro-Oncology. 2016; 18: 27-36.

[8] Pardridge WM. Blood-brain barrier drug delivery of IgG fusion proteins with a transferrin receptor monoclonal antibody. Exp Opin Drug Del. 2015; 12: 207-222.

[9] Neuwelt EA, Hill SA, Frenkel EP. Osmotic blood-brain barrier modification and combination chemotherapy: Concurrent tumor regression in areas of barrier opening and progression in brain regions distant to barrier opening. Neurosurgery. 1984; 15: 362-366.

[10] Deli MA. Potential use of tight junction modulators to reversibly open membranous barriers and improve drug delivery. Biochim Et Biophys Acta-Biomembranes. 2009; 1788: 892-910.

[11] Wong V, Gumbiner BM. A synthetic peptide corresponding to the extracellular domain of occludin perturbs the tight junction permeability barrier. J Cell Biol. 1997; 136: 399-409.

[12] Zwanziger D, Hackel D, Staat C, Bocker A, Brack A, Beyermann M, Rittner H, Blasig IE. A peptidomimetic tight junction modulator to improve regional analgesia. Mol Pharm. 2012; 9: 1785-1794.

[13] Raymond JJ, Robertson DM, Dinsdale HB. Pharmacological modification of bradykinin induced breakdown of the blood-brain barrier. Can J Neurol Sci. 1986; 13: 214-220.

[14] Makagiansar IT, Avery M, Hu Y, Audus KL, Siahaan TJ. Improving the selectivity of HAV-peptides in modulating E-cadherin-E-cadherin interactions in the intercellular junction of MDCK cell monolayers. Pharm Res. 2001; 18: 446-453.

[15] Sinaga E, Jois SD, Avery M, Makagiansar IT, Tambunan US, Audus KL, Siahaan TJ. Increasing paracellular porosity by E-cadherin peptides: discovery of bulge and groove regions in the EC1-domain of E-cadherin. Pharm Res. 2002; 19: 1170-1179.

[16] Tabanor K, Lee P, Kiptoo P, Choi IY, Sherry EB, Eagle CS, Williams TD, Siahaan TJ. Brain delivery of drug and MRI contrast agent: Detection and quantitative determination of brain deposition of CPT-Glu using LC-MS/MS and Gd-DTPA using magnetic resonance imaging. Mol Pharm. 2016; 13: 379-390.

[17] On NH, Kiptoo P, Siahaan TJ, Miller DW. Modulation of blood-brain barrier permeability in mice using synthetic E-cadherin peptide. Mol Pharm. 2014; 11: 974-981.

[18] Laksitorini MD, Kiptoo PK, On NH, Thliveris JA, Miller DW, Siahaan TJ. Modulation of intercellular junctions by cyclic-ADT peptides as a method to reversibly increase blood-brain barrier permeability. J Pharm Sci. 2015;104: $1065-1075$.

[19] Kiptoo P, Sinaga E, Calcagno AM, Zhao H, Kobayashi N, Tambunan US, Siahaan TJ. Enhancement of drug absorption through the blood-brain barrier and inhibition of intercellular tight junction resealing by E-cadherin peptides. Mol Pharm. 2011; 8: 239-249.

[20] Alaofi A, On N, Kiptoo P, Williams TD, Miller DW, Siahaan TJ. Comparison of Linear and Cyclic His-Ala-Val Peptides in Modulating the Blood-Brain Barrier Permeability: Impact on Delivery of Molecules to the Brain. J Pharm Sci. 2016; 105: 797-807.

[21] Osting S, Bennett A, Power S, Wackett J, Hurley SA, Alexander AL Agbandje-Mckena M, Burger C. Differential effects of two MRI contrast agents on the integrity and distribution of rAAV2 and rAAV5 in the rat striatum. Mol Ther Methods Clin Dev. 2014; $1: 4$.

[22] Xu CR, Yusuf-Makagiansar H, Hu Y, Jois SD, Siahaan TJ. Structural and ICAM-1-docking properties of a cyclic peptide from the I-domain of LFA-1: an inhibitor of ICAM-1/LFA- 1-mediated T-cell adhesion. J Biomol Struct Dyn. 2002; 19: 789-799.

[23] Yusuf-Makagiansar H, Siahaan TJ. Binding and internalization of an LFA-1-derived cyclic peptide by ICAM receptors on activated lymphocyte: A potential ligand for drug targeting to ICAM-1-expressing cells. Pharm Res. 2001; 18: 329-335.

[24] Anderson ME, Siahaan TJ. Mechanism of binding and internalization of ICAM-1-derived cyclic peptides by LFA- 1 on the surface of T cells: A potential method for targeted drug delivery. Pharm Res. 2003; 20: 1523-1532.

[25] Anderson ME Yakovleva $\mathrm{T}, \mathrm{Hu}, \mathrm{Y}$, Siahaan $\mathrm{TJ}$. Inhibition of ICAM-1/LFA-1-mediated heterotypic T-cell adhesion to epithelial cells: design of ICAM-1 cyclic peptides. Bioorg Med Chem Lett. 2004; 14: 1399-1402.

[26] Zimmerman T, Oyarzabal J, Sebastian ES, Majumdar S, Tejo BA, Siahaan TJ, Blanco FJ. ICAM-1 peptide inhibitors of T-cell adhesion bind to the allosteric site of LFA-1. An NMR characterization. Chem Biol Drug Des. 2007; 70: 347-353.

[27] Triguero D, Buciak J, Pardridge WM. Capillary depletion method for quantification of blood-brain barrier transport of circulating peptides and plasma proteins. J Neurochem. 1990; 54: 1882-1888.

[28] Bella J, Kolatkar PR, Marlor CW, Greve JM, Rossmann MG. The structure of the two amino-terminal domains of human ICAM-1 suggests how it functions as a rhinovirus receptor and as an LFA-1 integrin ligand. Proc Natl Acad Sci U S A. 1998; 95: 4140-4145.

[29] Yusuf-Makagiansar H, Makagiansar IT, Siahaan TJ. Inhibition of the adherence of T-lymphocytes to epithelial cells by a cyclic peptide derived from inserted domain of lymphocyte function-associated antigen-1. Inflammation. 2001; 25: 203-214.

[30] Anderson ME, Tejo BA, Yakovleva T, Siahaan TJ. Characterization of binding properties of ICAM-1 peptides to LFA-1: inhibitors of T-cell adhesion. Chem Biol Drug Des. 2006; 68: 20-28.

[31] Majumdar S, Kobayashi N, Krise JP, Siahaan TJ. Mechanism of internalization of an ICAM-1-derived peptide by human leukemic cell line HL-60: Influence of physicochemical properties on targeted drug delivery. Mol Pharm. 2007; 4: 749-758.

[32] Services DoHaH, Administration FaD, (CDER) CfDEaR, (CBER) CfBEaR. Analytical Procedures and Methods Validation for Drugs and Biologics: Guidance for Industry. Pharmaceutical Quality/CMC. 2015;: 1-15.

[33] Alaofi A, Farokhi E, Prasasty VD, Anbanandam A, Kuczera K, Siahaan TJ. Probing the interaction between $\mathrm{CHAVc} 3$ peptide and the EC1 domain of E-cadherin using NMR and molecular dynamics simulations. J Biomol Struct Dyn. 2017; 35: 92-104.

[34] Rubin LL, Staddon JM. The cell biology of the blood-brain barrier. Annu Rev Neurosci. 1999; 22: 11-28.

[35] Pal D, Audus KL, Siahaan TJ. Modulation of cellular adhesion in bovine brain microvessel endothelial cells by a decapeptide. Brain Res. 1997; 747: 103-113.

[36] Abbruscato TJ, Davis TP. Protein expression of brain endothelial cell E-cadherin after hypoxia/aglycemia: influence of astrocyte contact. Brain Res. 1999; 842: 277-286.

[37] Vorbrodt AW, Dobrogowska DH. Molecular anatomy of interendothelial junctions in human blood-brain barrier microvessels. Folia Histochem Cytobiol. 2004; 42: 67-75.

[38] Vorbrodt AW, Dobrogowska DH. Molecular anatomy of intercellular junctions in brain endothelial and epithelial barriers: Electron microscopist's view. Brain Research Reviews. 2003; 42: 221-242.

[39] Lutz KL, Siahaan TJ. Modulation of the cellular junction protein E-cadherin in bovine brain microvessel endothelial cells by cadherin peptides. Drug Delivery. 1997; 4: 187-193.

[40] Boggon TJ, Murray J, Chappuis-Flament S, Wong E, Gumbiner BM, Shapiro L. C-cadherin ectodomain structure and implications for cell adhesion mechanisms. Science. 2002; 296: 1308--1313. 